\title{
UTILIZAÇÃO DA APRENDIZAGEM BASEADA EM PROJETO (ABP) PARA O DESENVOLVIMENTO DE UM ROBÔ AUTÔNOMO PARA MAPEAMENTO DE AMBIENTES CONTROLADOS
}

Catherine M. de Freitas - catherine.freitas@ sou.unijui.edu.br

Pedro G. Pascoal - pedro.pascoal@ sou.unijui.edu.br

Victor N. Kürschner - vickurschner@ hotmail.com

Universidade Regional do Noroeste do Estado do Rio Grande do Sul - UNIJUÍ, Departamento de Ciências Exatas e Engenharias - DCEEng,

Rua Lulu Ilgenfritz, Bairro São Geraldo, nº 480,

98700-000 - Ijuí - Rio Grande do Sul.

Resumo: $O$ presente trabalho se refere ao desenvolvimento de um robô autônomo com a capacidade de mapear ambientes internos controlados. $O$ objetivo do projeto foi realizar a aplicação prática dos conhecimentos adquiridos na disciplina de robótica através do método de Aprendizado Baseado em Projeto, onde os estudantes aprimoraram suas habilidades referentes a resolução de problemas tecnológicos reais. Esse modelo de ensino ainda proporcionou a cooperação e envolvimento de todos os alunos, resultando em maior desempenho, bem como em um produto final satisfatório. Os alunos foram instigados a desenvolver o projeto aplicando materiais e métodos distintos dos estudados durante a graduação. O robô desenvolvido é capaz de se movimentar de maneira autônoma além de enviar dados de distância de obstáculos para um dispositivo móvel através de comunicação wireless.

Palavras-chave: Ensino. Microcontrolador. Multidisciplinaridade. Robótica.

\section{INTRODUÇÃO}

A Aprendizagem Baseada em Projetos (ABP) é uma metodologia de ensino ativa que promove experiências de aprendizagem significativas aos alunos. Nessa metodologia os participantes têm a oportunidade de construir o conhecimento resolvendo problemas reais com a finalidade de desenvolver um produto final. Para alcançar os objetivos propostos por essa metodologia é necessário o envolvimento por parte dos alunos e também dos professores, que devem motivar, orientar e apoiar os alunos no decorrer das atividades. A colaboração e empenho dos estudantes promove, além da concretização das ideias em um produto físico, o desenvolvimento de habilidades como a criatividade, a autossuficiência e trabalho colaborativo. Nesse contexto, os alunos são direcionados a compreensão de como suas habilidades podem ser empregadas em atividades relacionadas a sua futura área de atuação (KOKOTSAKI, MENZIES, WIGGINS, 2016).

A robótica é uma área multidisciplinar que permite a integração de conhecimentos referentes a eletrônica, instrumentação, controle, automação etc. (CAMPOS et al., 2018). Além disso é uma área de interesse tanto dos alunos como da indústria. A capacidade de navegar em um ambiente de forma independente, é um recurso relevante para robôs móveis (HENTSCHEL E WAGNER, 2010). O processo de navegação autônoma consiste, 
basicamente, em captar dados do ambiente e definir sua rota sem a necessidade de interferência humana. Sistemas de monitoramento autônoma para o mapeamento de ambientes apresentam aplicações domésticas, industriais, militares e urbanas sendo de grande interesse o desenvolvimento de pesquisas nessa área (SAUTHIER, 2016).

De acordo com CAMPOS et al. (2018) a criação de uma máquina autônoma, requer a aplicação de conhecimentos que ultrapassam os domínios da eletrônica, sendo necessário também, habilidades referentes a matemática, mecânica, física e o design. Nesse sentido, o presente trabalho apresenta a utilização da metodologia ABP para o desenvolvimento de um robô autônomo com mapeamento de obstáculos. Ele deve se movimentar em um ambiente controlado de forma independente através do uso de sensores e lógica de programação. Os dados de posicionamento dos objetos e do robô são enviados a dispositivos móveis através de protocolo de comunicação sem fío. O robô foi desenvolvido para a disciplina de robótica do curso de Engenharia Elétrica, com o intuito de promover a aplicação de conhecimentos multidisciplinares.

O trabalho está organizado em 6 seções, sendo abordado na seção 2 os materiais e métodos utilizados para o desenvolvimento do robô. Em seguida, na seção 3, é apresentado o circuito de acionamento dos atuadores. Posterior ao projeto dos circuitos são comentadas na seção 4, as lógicas de programação relacionadas aos códigos desenvolvidos para os microcontroladores implementados no projeto. A seção 5 contém as placas de circuito impresso e a interface para visualização dos dados de sua posição. E, por fim, na seção 6 são apresentadas as considerações finais.

\section{MATERIAIS E MÉTODOS}

Este capítulo tem a finalidade de apresentar os aspectos estruturais que são relacionados a mobilidade do robô. Para isso, são utilizadas 4 rodas tracionadas e controladas de modo independente por 4 motores de corrente contínua (CC). A base de acoplamento das demais estruturas é confeccionada por uma chapa metálica, contendo uma caixa para abrigar os circuitos eletrônicos. A fim de detectar os obstáculos é utilizado um sensor de distância localizado sobre o eixo de um servomotor que amplia o campo de visão do robô. A informação do sensor é processada e enviada através de microcontroladores (MCU).

\subsection{Atuadores}

Os motores de corrente contínua implementados no projeto são o modelo TFK280SC21138-45. Eles possuem tensão nominal de 7,2 V, corrente a vazio e máxima de $160 \mathrm{~mA}$ e 2,5 A, respectivamente. O eixo dos motores é acoplado a uma caixa de redução $(86,8: 1)$, permitindo a atenuação da sua velocidade para, aproximadamente, 100 RPM, desenvolvendo um torque de $10 \mathrm{kgf.cm}(0,98$ N.m).

A escolha dos motores ocorreu devido a sua disponibilidade no laboratório além de proporcionarem um torque elevado se comparado aos demais motores disponíveis. Outra característica interessante, é a presença de encoders de quadratura internos que permitem a futura implementação de controle de velocidade. A partir da definição dos atuadores, foram selecionadas as rodas disponíveis em laboratório. Sendo assim, foram utilizadas rodas confeccionadas de plástico e borracha com eixo metálico, que possuem diâmetro de $13 \mathrm{~cm}$. Elas foram acopladas diretamente aos eixos dos atuadores.

\subsection{Sensor de distância}

Para que o robô identifique a presença de obstáculos, é proposta a utilização do sensor de distância SHARP GP2Y0A02YK0F. Ele faz medições de $20 \mathrm{~cm}$ a $150 \mathrm{~cm}$ através de ondas 
infravermelhas, com ângulo de abertura equivalente a distância entre o emissor e o receptor. O sensor SHARP não sofre com interferências externas, como temperatura e cores dos objetos, possuindo precisão elevada. Ele contém um circuito de aquisição de sinais disponibilizando três terminais para ligação, dois referentes a alimentação de $5 \mathrm{~V}$ e um de sinal de saída. O terminal de saída de dados é representado por uma tensão analógica que varia conforme a distância mensurada, de acordo com a curva característica disponibilizada pelo fabricante.

Com o intuito de ampliar o campo de visão do robô, o sensor foi acoplado sobre o eixo de um servomotor modelo SG90, que possui $180^{\circ}$ de liberdade. O ângulo do servomotor é controlado através da largura de pulso de um sinal de onda retangular de $50 \mathrm{~Hz}$.

\subsection{Microcontroladores}

Para o desenvolvimento das lógicas de programação foram utilizados dois microcontroladores, o STM32F103C8 e o ESP8285. O primeiro é responsável pelas funções de controle dos motores e medições do sensor. É um MCU de 32 bits, baseado na arquitetura ARM (Advanced RISC Machine), podendo trabalhar com uma frequência de até $72 \mathrm{MHz}$, possui 64 kbytes de memória Flash e 2 kbytes de memória RAM. Também contém dois conversores A/D (Analógico/Digital) de 12 bits, um controlador DMA (Direct Memory Acess), 4 timers e compatibilidade com protocolos de comunicação, como SPI, I²C e UART.

O STM32F103C8 realiza o processamento de dados provenientes do sensor e transmite para o MCU ESP8285 através do protocolo de comunicação serial UART. O ESP é responsável por transmitir esses dados de posicionamento a um dispositivo móvel. A programação e depuração do código podem ser realizadas com comandos AT, ou através do método Standalone. Apesar de ser um microcontrolador com capacidade de sintetizar um sistema de comunicação Wi-Fi próprio, o ESP8285 pode servir como módulo auxiliar para outros MCU que não possuem capacidade de conexão Wi-Fi (PASCOAL, 2020).

Nesse trabalho o ESP foi utilizado como um módulo auxiliar do SMT32F103C8, ele é configurado para receber dados via comunicação serial e os transferir para uma página Web com um IP definido, os sinais são enviados utilizando a antena impressa na própria placa, com frequência de 2,4 GHz.

\subsection{Alimentação}

Para o funcionamento do robô, são necessários 4 níveis de tensão que estão descritos na Quadro 1. Para isso, foi desenvolvido um pack reutilizando baterias de Lítio de notebook. A capacidade de cada célula é de $2200 \mathrm{mAh}$, possuindo tensão nominal de 3,7 V e máxima de 4,2 V com 3,0 V de nível de cutoff. São utilizadas um total de 6 baterias em uma associação série/paralelo de forma a fornecer 2 níveis de tensão distintos, 7,4 V e 11,1 V nominais. Uma imagem ilustrativa da ligação feita é apresentada na Figura 1.

Quadro 1 - Níveis de tensão
\begin{tabular}{|c|c|}
\hline Nível de Tensão & Componentes \\
\hline $12,0 \mathrm{~V}$ & LEDs \\
\hline $7,2 \mathrm{~V}$ & Motores CC \\
\hline $5,0 \mathrm{~V}$ & Sensor e Servomotor \\
\hline $3,3 \mathrm{~V}$ & Microcontroladores \\
\hline
\end{tabular}

Fonte: Autores (2020) 
Figura 1 - Ligação do pack de baterias

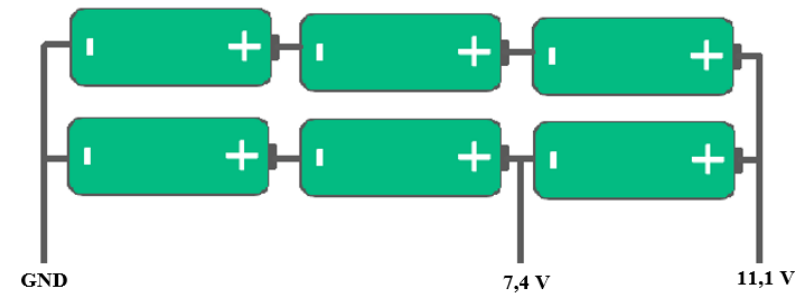

Fonte: Autores (2020)

Para obtenção dos 5,0 V de alimentação do sensor, é utilizado o regulador linear L7805AB2T com sua entrada ligada aos $7,4 \mathrm{~V}$. Os $5,0 \mathrm{~V}$ regulados são utilizados para alimentar a placa de desenvolvimento do microcontrolador, o qual já possui um regulador de 3,3 V embarcado para alimentar o circuito integrado.

\subsection{Projeto e montagem da estrutura}

A estrutura do robô foi projetada utilizando o software Fusion 360 da Autodesk. A base para acoplamento dos componentes é composta por uma chapa metálica retangular com cantos arredondados, extremidades curvas e dimensões aproximadas de $18,5 \mathrm{~cm}$ x $12,5 \mathrm{~cm}$. Os motores e as baterias foram dispostos na parte inferior da base, e o sensor, bem como os circuitos de controle e acionamento foram posicionados em sua parte superior.

A fim de abrigar os circuitos de controle e acionamento foi projetada uma caixa, na qual foram fixadas, na parte interna, os circuitos eletrônicos. O sensor de distância, junto ao servomotor, foi posicionado em frente a caixa, no seu lado externo, conforme a Figura 2, que apresenta o projeto tridimensional do robô.

Figura 2 - Simulação 3D da estrutura
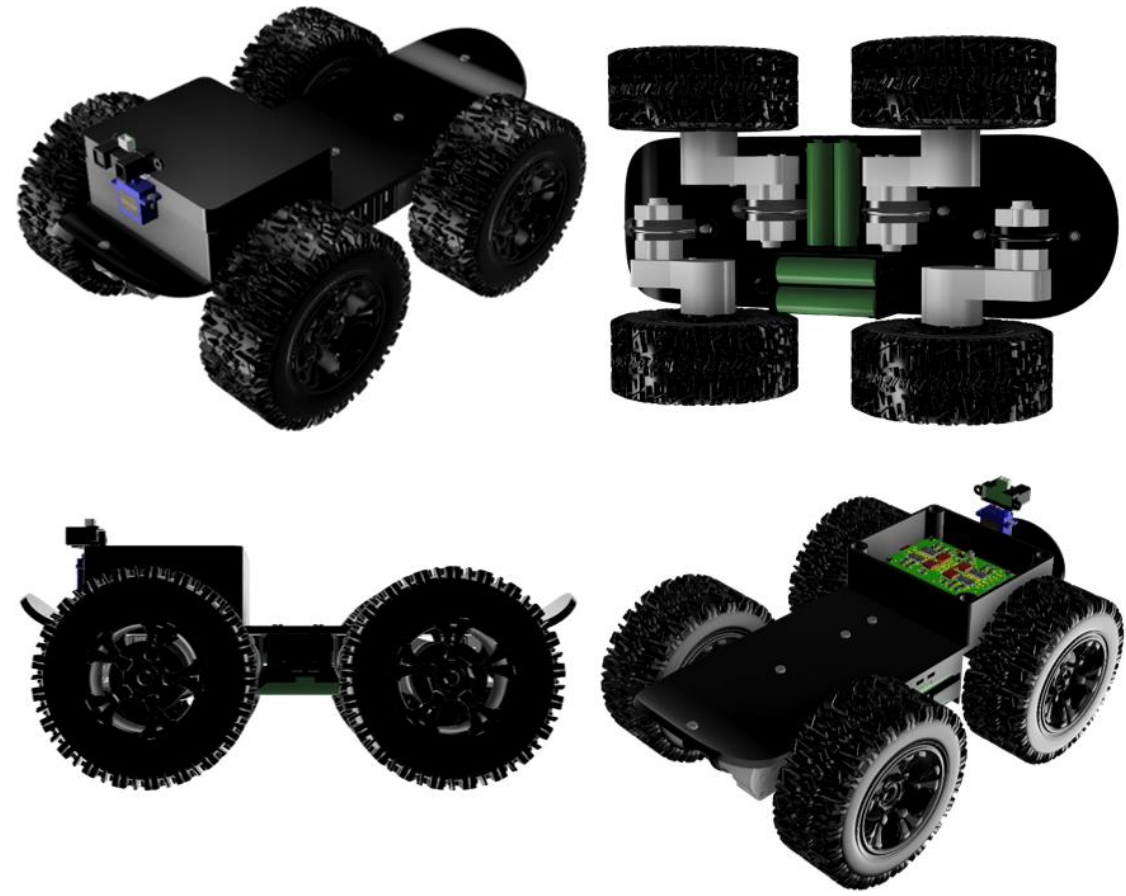

Fonte: Autores (2020)

A partir do projeto foi executada a estrutura, que pode ser visualizada na Figura 3. 


\section{COBENGE C.COBENCE 2020 da ABENGE}

"Os desafios para formar hoje o engenheiro do amanhã"

Figura 3 - Vistas inferior e superior do robô

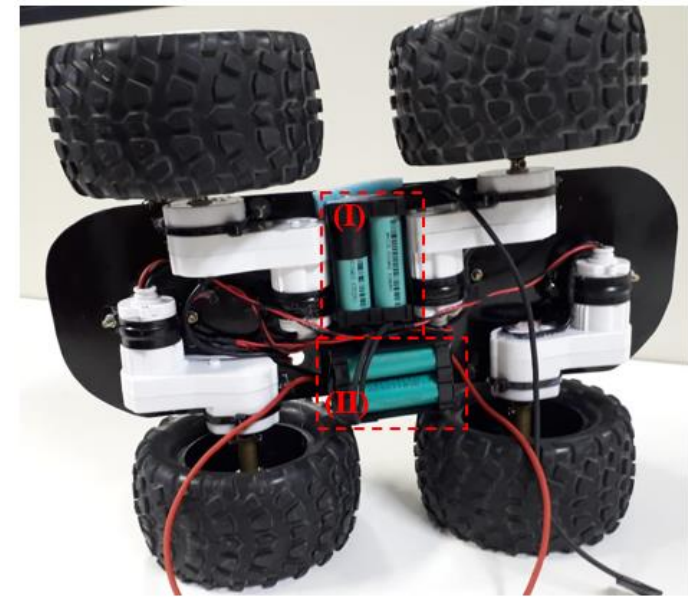

(a)

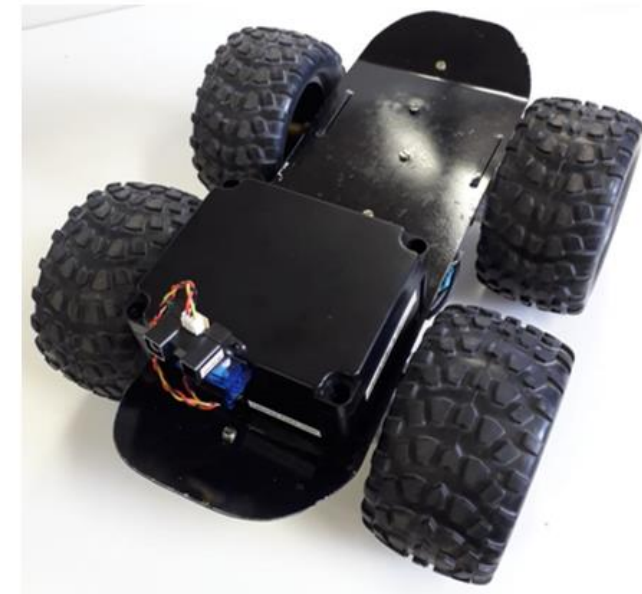

(b)

Fonte: Autores (2020)

A disposição dos motores e das baterias na estrutura podem ser analisadas na Figura 3 (a), onde os conjuntos I e II contêm 2 e 4 baterias, respectivamente. $\mathrm{O}$ circuito de controle do robô, bem como o circuito de acionamento dos motores são fixadas no interior de uma caixa da marca Patola de modelo PBL 120 cujas dimensões são $10 \mathrm{~cm}$ x $12 \mathrm{~cm}$ x $5 \mathrm{~cm}$. A Figura 3 (b) apresenta a localização da caixa, do sensor e do servomotor.

\section{ACIONAMENTO DOS ATUADORES}

Este capítulo trata sobre o circuito de acionamento dos atuadores, que foi elaborado após as definições dos principais materiais componentes do projeto descritos.

Para realizar o controle de sentido de giro dos motores é necessário um circuito driver (ponte $\mathrm{H}$ ) capaz de inverter o fluxo de corrente que circula pelos seus terminais (MEHTA et al., 2014). A topologia mais simples desse circuito é composta de quatro chaves eletrônicas, conforme Figura 5, que são acionadas de formas opostas, ou seja, S1 e S4, ou S2 e S3. Logo, a diferença entre essas duas formas de acionamento faz o robô se movimentar para frente ou para trás.

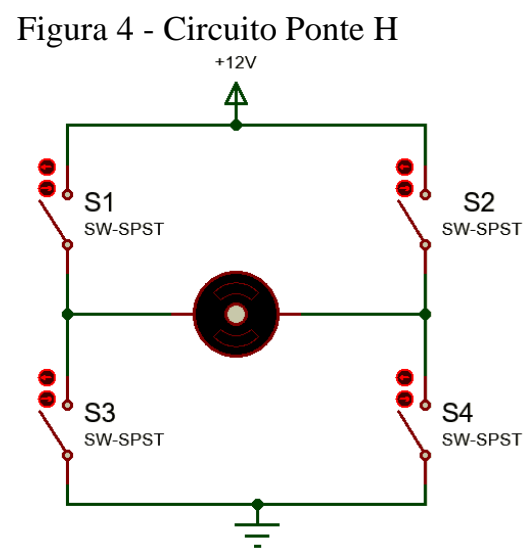

Fonte: Autores (2020)

Cada lado do robô é composto por dois motores, assim, a demanda de corrente máxima por canal é de 5,0 A. Dessa forma, as chaves eletrônicas devem ser capazes de suportar as condições exigidas. Nesse trabalho são utilizados os MOSFETs FDD6637 (canal P) e 
FDD8796 (canal N) que, de acordo com sua documentação, atendem as necessidades de projeto, fornecendo uma resistência interna reduzida, que minimiza as perdas por aquecimento.

Com o intuito de controlar a tensão e, consequentemente a velocidade dos motores, é utilizado o método Pulse Width Modulation (PWM). O PWM é um sinal de onda quadrada que ao ser aplicado no MOSFET realiza o controle de velocidade do motor através do duty cycle, que representa em porcentagem, quanto tempo a chave fica acionada. Essa forma de onda é gerada com o microcontrolador STM32F103C8T6 o qual fornece uma corrente máxima de $25 \mathrm{~mA}$.

O MOSFET possui uma capacitância intrínseca a qual deve ser carregada e descarregada com uma corrente superior aos $25 \mathrm{~mA}$ fornecidos pelo microcontrolador. Dessa forma, é necessária a utilização de um circuito de acionamento para que a carga e descarga desse capacitor seja feita através da fonte de alimentação do circuito, e não pelo STM32F103C8T6. Para isso são utilizados optoacopladores, modelo 6N137, que isolam o sinal do microcontrolador e o inserem em um driver denominado Push-Pull, Figura 6. Esse circuito é composto por transistores bipolares de junção (TBJ) FCX690BTA (NPN) e o BC557B (PNP).

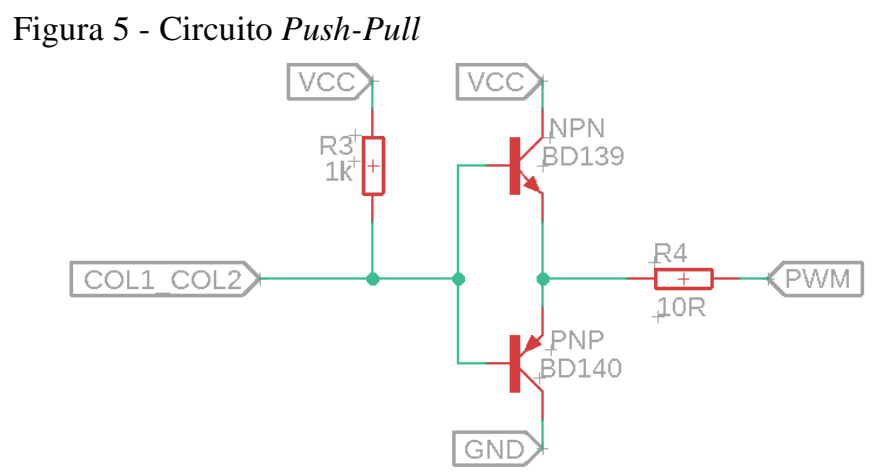

Fonte: Autores (2020)

\section{CÓDIGOS DE PROGRAMAÇÃO}

Posterior ao projeto dos circuitos de acionamento dos atuadores, tornaram-se necessários os sinais de chaveamento dos semicondutores, além da lógica de programação para o controle da movimentação do robô a partir dos dados provenientes do sensor de distância. Sendo assim, nessa seção serão comentadas as lógicas de programação relacionadas aos códigos desenvolvidos para os microcontroladores implementados no projeto.

\subsection{Microcontrolador ARM}

A programação do MCU STM32F103C8 foi inicializada através da configuração dos seus periféricos. O timer 2 foi destinado exclusivamente para produzidos os PWMs de acionamento dos motores, cujas frequências são de $2 \mathrm{kHz}$. O timer 3 foi configurado para realizar toda a lógica de controle do robô, ou seja, a partir dele são definidos os tempos de todas as demais funções. Dentre elas podem ser encontradas a medição referentes a leitura do canal A/D - que ocorre a cada dois ciclos desse timer para respeitar o tempo de leitura de 39 ms do SHARP -, além do sinal PWM com frequência de $50 \mathrm{~Hz}$ para o servomotor, onde é permitindo a variação do duty cycle através das interrupções de tempos fixos a cada $20 \mathrm{~ms}$.

A lógica de controle implementada no código consiste em realizar a movimentação do robô para frente - enquanto não haja obstáculos a uma distância menor que $40 \mathrm{~cm}$ - e a leitura do sensor, que ocorrem simultaneamente. Caso ocorra a verificação de um objeto posicionado 
a direita do robô (a menos de $40 \mathrm{~cm}$ ) são acionados os motores de modo que ele siga na direção oposta (esquerda). Seu ângulo de giro é definido em relação a maior leitura de distância do sensor. Essa análise ocorre da mesma forma para a detecção de obstáculos à esquerda. Com o intuito de eliminar a possibilidade de o robô entrar em um loop, ou seja, não seguir para nenhuma das direções estando "preso" em determinado espaço, foi implementada uma lógica de ré para que ele retornar à posição anterior e definir novamente sua rota e rotina de mapeamento.

Durante a rotina de mapeamento é desenvolvido o monitoramento do nível de tensão fornecido pelo pack de baterias. Essa verificação é realizada constantemente através de um conversor $\mathrm{A} / \mathrm{D}$ que recebe o sinal de tensão atenuado e proporcional à sua capacidade máxima. Dessa forma, se a indicação de tensão for inferior a $50 \%$, os LEDs fixados nos motores oscilam informando o usuário sobre a necessidade de carregamento.

As informações de posicionamento referentes ao robô e os objetos detectados pelo sensor SHARP são processadas no STM32F103C8 e transmitidas ao ESP8285 através do protocolo de comunicação UART. Essa interface permite o envio e recebimento de dados de forma assíncrona através dos terminais transmissor $(\mathrm{Tx})$ e receptor $(\mathrm{Rx})$.

\subsection{Microcontrolador ESP}

O código do MCU ESP8285 foi desenvolvido a partir da função de recebimento de dados do ARM. Sendo assim, a lógica de programação implementada inicia com a verificação de integridade dessas informações, que, posteriormente, são atribuídas a um vetor com capacidade armazenar 19 valores de posição distintos.

Com o intuito de transmitir os dados para um Web Server, o ESP foi conectado à rede Wi-Fi local gerando automaticamente um endereço IP. A interface gráfica do Web Server foi desenvolvida através das linguagens de programação HTML, Javascript e CSS. Sendo assim, quando ocorre a inicialização do $\mathrm{MCU}$, já é realizada a requisição da página $W e b$, bem como dos dados de posição armazenados, que são enviados às suas respectivas posições no gráfico resultante. Os valores de posição recebem atualizações a cada segundo.

\section{RESULTADOS}

Nessa seção serão expostos os resultados provenientes do estudo, projeto e desenvolvimento do robô a partir do método ABP abordado para a elaboração do trabalho. Dessa forma, são apresentadas as placas de circuito (PCI) impresso referentes ao acionamento dos motores e ao controle das demais funções do robô, além da interface para visualização dos dados de sua posição.

\subsection{Placa de acionamento dos atuadores}

Para o circuito de acionamento dos motores foi projetada uma placa de circuito impresso no software EAGLE da Autodesk. A Figura 5 (a) apresenta a placa com a perspectiva presente no software, bem como a versão final da montagem prática dos componentes em (b).

Os motores são acionados em pares (lado direito e esquerdo), logo, a PCI possui dois lados de controle, onde os motores são conectados em paralelo. O circuito foi desenvolvido com componentes SMD (Surface Mount Device), possibilitando a redução do tamanho final da placa, resultando em dimensões de $8,5 \mathrm{~cm}$ x $5,5 \mathrm{~cm}$. Para facilitar as conexões com os motores e com o banco de baterias foram inseridos conectores de $90^{\circ}$, que também colaboram no acesso para manutenção. 


\section{COBENCE de Educação em Engenharia da ABENGE}

"Os desafios para formar hoje o engenheiro do amanhã"

Figura 6 - Placa de circuito impresso da Ponte H
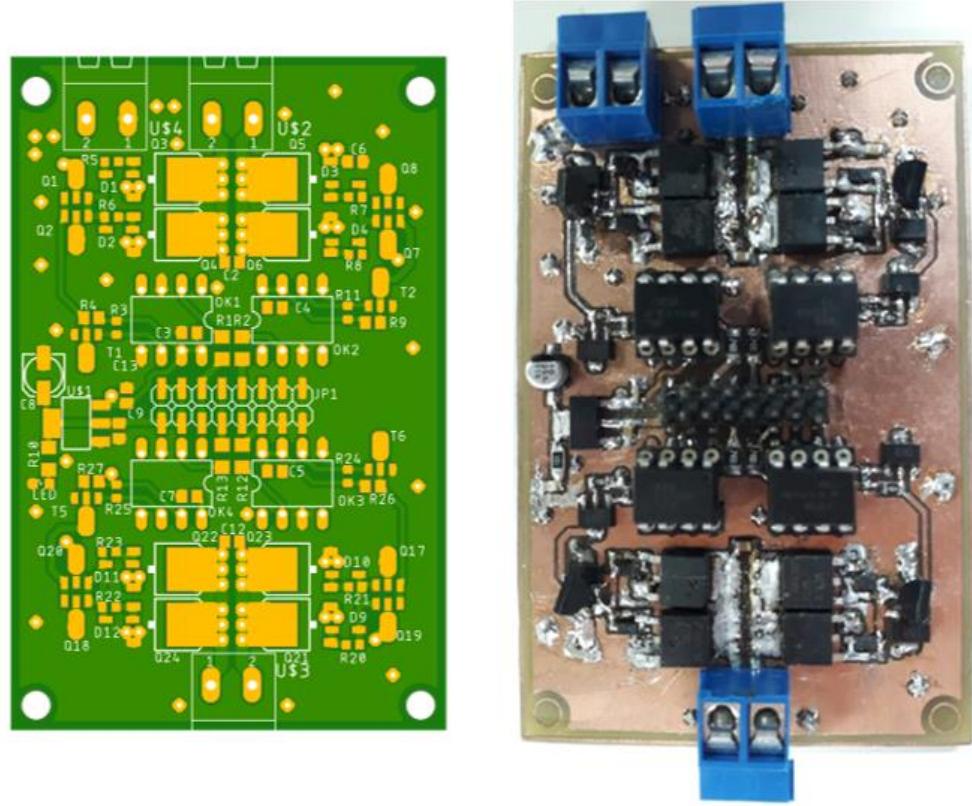

Fonte: Autores (2020)

Os terminais de conexão presentes na parte central dessa PCI são destinados para conexão com a placa de controle.

\subsection{Placa de controle}

Para o controle do robô foi projetada outra placa de circuito impresso, também desenvolvida no software EAGLE. Ela pode ser observada na Figura 6 (a) e (b).
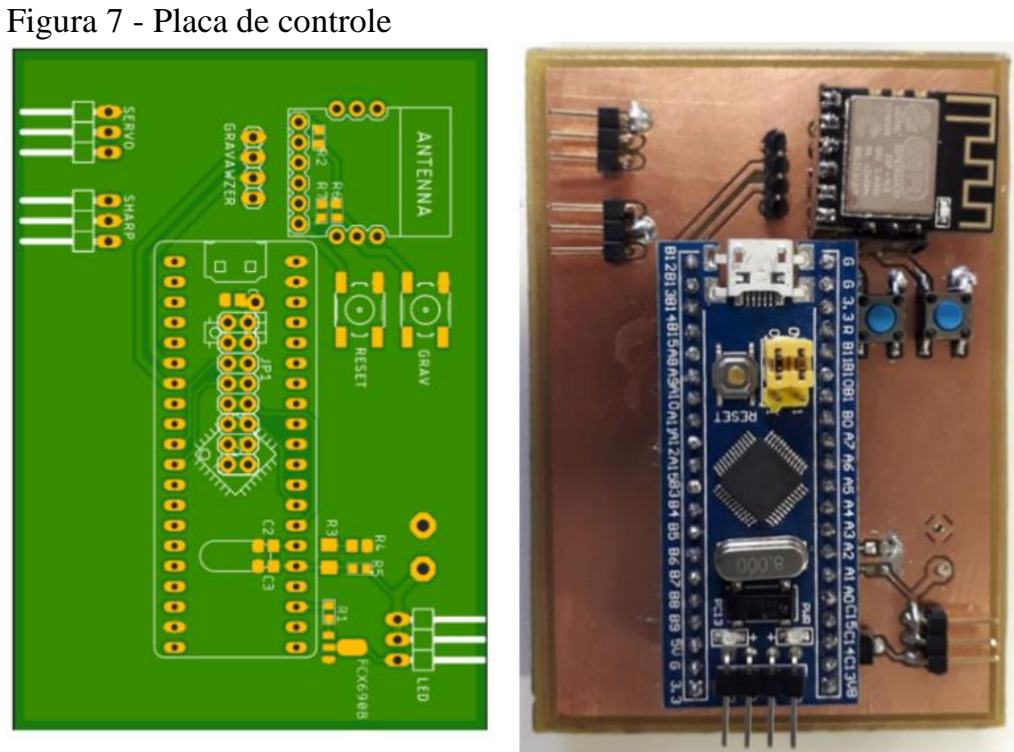

Fonte: Autores (2020)

Essa placa é composta, basicamente, pelos microcontroladores STM32F103C8 e ESP8285, além de possuir conectores para ligação com o servomotor, além do sensor SHARP e dos LEDs. As dimensões da PCI são de $6,0 \mathrm{~cm} \mathrm{x} 8,5 \mathrm{~cm}$, e ela possui um conector complementar ao da placa de acionamento dos motores, que possibilita seu encaixe e transferência de informações mantendo os dois circuitos na parte interna da caixa protetora. 


\subsection{Interface Web}

A partir do projeto e confecção da estrutura do robô, bem como da alocação dos seus componentes, como as placas de circuito impresso e o desenvolvimento dos códigos de programação, foi possível a comunicação e envio de dados referentes a posição do protótipo.

Como comentado no capítulo anterior, o Web Server foi desenvolvido a partir das linguagens de programação HTML, Javascript e CSS, e pode ser visualizado na Figura 7.

Figura 8 - Interface de posicionamento do robô

\begin{tabular}{|c|c|}
\hline$\Delta 0$ & (1) $>\boldsymbol{\Delta}=12: 13$ \\
\hline$\times \quad$ (i) $\begin{array}{l}192.168 .137 .221 \\
192.168 .137 .221\end{array}$ & 口 \\
\hline
\end{tabular}

\section{Posicionamento do Robo}

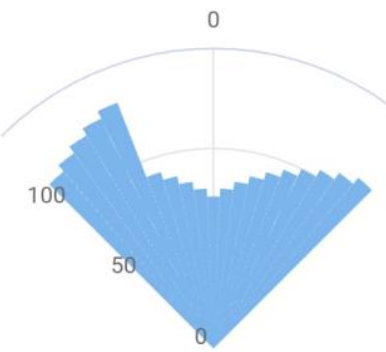

Fonte: Autores (2020)

A partir da Figura 7 é perceptível a presença de um gráfico que possui valores de distância relacionados à posição do robô com os obstáculos detectados à sua frente. Esses valores são interpretados em tempo real pelo sensor SHARP e, após processados pelos MCUs, são requisitados pela página com atualizações a cada segundo, o que permite o operador acompanhar a localização dos obstáculos e os movimentos do protótipo.

\section{CONSIDERAÇÕES FINAIS}

O trabalho tratou da aplicação da metodologia de aprendizagem baseada em projeto para o desenvolvimento de um robô autônomo com mapeamento de obstáculos. Esse robô foi desenvolvido no período da disciplina de robótica que instigou a criatividade dos alunos. Além do projeto e confecção da estrutura do protótipo, foram desenvolvidos códigos de programação em diferentes microcontroladores a fim de controlar sua locomoção e monitorar o espaço ao qual ele está inserido. Posterior a isso, as informações referentes às distâncias entre o robô e os obstáculos encontrados foram enviadas a um Web Server criado dentro da rede Wi-Fi local - com um endereço IP específico -, que demonstra os dados do sensor medidor de distância em um gráfico.

A aplicação da metodologia ABP permitiu a utilização de conhecimentos interdisciplinares exigidos pela área da robótica, promovendo o desenvolvimento de habilidades essenciais a um profissional da Engenharia Elétrica. Além de instigar a busca por experiências que não foram abordadas no curso/currículo original a fim de alcançar soluções concretas.

A estrutura do método foi eficaz, possibilitando os estudantes trabalharem em equipe aprofundando seus conhecimentos, além de pesquisarem soluções imediatas e acessíveis a partir de componentes disponibilizados pela infraestrutura da universidade. 
Dessa forma, a ABP orientou a buscar o novo, usar a criatividade e lidar com pressões, prazos e orçamentos limitados, que são característicos de projetos desta natureza. Tornando perceptível a contribuição do trabalho para a formação dos futuros profissionais, com maior qualificação para o mercado.

\section{Agradecimentos}

Os autores agradecem a instituição de ensino UNIJUÍ, ao Grupo de Automação Industrial e Controle (GAIC) e ao grupo de pesquisa Física para Todos pela disponibilidade da infraestrutura e equipamentos necessários para o desenvolvimento do projeto.

\section{REFERÊNCIAS}

CAMPOS, M. et al. PBL: A Utilização de Aprendizagem Baseada em Projeto para o Desenvolvimento de um Robô Interativo com Reconhecimento Facial. XXII Congresso Brasileiro de Automática. 2018.

HENTSCHEL, M.; WAGNER, B. Autonomous robot navigation based on openstreetmap geodata. International IEEE Conference on Intelligent Transportation Systems. IEEE, 2010.

KOKOTSAKI, D.; MENZIES, V.; WIGGINS, A. Project-based learning: A review of the literature. Improving schools, v. 19, n. 3, p. 267-277, 2016.

MEHTA, K. et al. Design implementation of high performance DC motor drive. 2014 International Conference on Advances in Computing, Communications and Informatics (ICACCI). IEEE, 2014.

PASCOAL, P. G. Desenvolvimento de um Sistema para Monitoramento da Frequência Cardíaca em Atividades Físicas. Trabalho de Conclusão de Curso. Universidade Regional do Noroeste do Estado do Rio Grande do Sul - UNIJUÍ, Ijuí, 2020.

SAUTHIER, L. F. Projeto e Desenvolvimento de um SONAR para Mapeamento Bidimensional de Ambientes Internos. 2016. Trabalho de Conclusão de Curso. Universidade Regional do Noroeste do Estado do Rio Grande do Sul - UNIJUÍ, Ijuí, 2016.

\section{USE OF PROJECT-BASE LEARNING (PBL) FOR THE DEVELOPMENT OF AN AUTONOMOUS ROBOT FOR MAPPING CONTROLLED ENVIRONMENTS}

Abstract: The present work refers to the development of an autonomous robot with the ability to map controlled indoor environments, with the objective of carrying out the practical application of the knowledge acquired in the discipline of robotics, through the Project Based Learning method, where students improved their skills concerning the resolution of real technological problems. This teaching model also provided the cooperation and involvement of all students, resulting in greater performance, as well as a satisfactory final product. Students were encouraged to develop the project using different materials and methods from those studied during graduation. The developed robot is able to move autonomously in addition to sending obstacle distance data to a mobile device via wireless communication.

Keywords: Teaching, Microcontroller, Multidisciplinarity, Robotics. 